\title{
The Potential of Nanomaterials for Drug Delivery, Cell Tracking, and Regenerative Medicine
}

\author{
Krasimir Vasilev, ${ }^{1,2}$ Haifeng Chen, ${ }^{3}$ and Patricia Murray ${ }^{4}$ \\ ${ }^{1}$ Mawson Institute, University of South Australia, Mawson Lakes, SA 5095, Australia \\ ${ }^{2}$ School of Advanced Manufacturing, University of South Australia, Mawson Lakes, SA 5095, Australia \\ ${ }^{3}$ Department of Biomedical Engineering, College of Engineering, Peking University, Beijing 100871, China \\ ${ }^{4}$ School of Biological Sciences, University of Liverpool, Liverpool L69 7ZB, UK \\ Correspondence should be addressed to Krasimir Vasilev, krasimir.vasilev@unisa.edu.au
}

Received 27 May 2012; Accepted 27 May 2012

Copyright (c) 2012 Krasimir Vasilev et al. This is an open access article distributed under the Creative Commons Attribution License, which permits unrestricted use, distribution, and reproduction in any medium, provided the original work is properly cited.

Recent progress in bio- and nanotechnology has led to the development of a rich variety of novel materials, precisely engineered at the nanoscale, which hold great potential to revolutionize traditional medical treatments and therapies. This special issue brings together and highlights the latest advances in the use of nanomaterials in biomedical technologies, such as drug delivery, cell tracking, imaging, and regenerative medicine as a whole. Regenerative medicine has been a rapidly developing field which is forecast to gain further momentum through growing government and commercial investments in R\&D and new technologies. Although emerging therapies need to undergo the inevitable regulatory approval processes, recent market reports suggest that, in the next five years, regenerative medicine will occupy a significant share of the biomedical space. Undoubtedly, the success of regenerative medicine would not have been possible without novel nanomaterials, including, but not limited to the following: nanoparticles, nanotubes, nanoengineered scaffolds, and nanoscale surface modifications. One of the aims of this special issue is to highlight the current challenges and obstacles which lie on the path to fully integrating these promising nanomaterial-based technologies in medicine. Further, this special issue aims to provide guidance to researchers in the field of bionanotechnology and to inform the research, medical, and industry communities of the current and future directions of the field.

This special issue is a collection of quality contributions from established laboratories around the world. It contains instructive review articles and original research articles dealing with diverse areas of regenerative medicine.
Seven excellent reviews published in this special issue present a critical overview of recent progress in the corresponding field of regenerative medicine and the authors' view for their future directions. Two of the reviews focus on drug delivery through hydrogels and porous silicon. A review article by S. Dey and T. K. Maiti discusses gene silencing though superparamagnetic nanoparticles. The progress and challenges of cell tracking and imaging with nanocrystals and nanoparticles used in magnetic resonance are discussed in two separate reviews. A paper by the group of K. Ostrikov draws the reader's attention to potential toxicity of novel nanomaterials such as grapheme and carbon nanotubes which hold great promise in many advanced applications including medicine. Recent advances in microfluidic platforms for evaluation of nanobiomaterials have also been brought to the attention of the readers of this special issue.

The special issue also contains 18 original research articles which make substantial contributions to various areas of regenerative medicine. These areas include the delivery of therapeutics, such as proteins and genes, through nanomaterials or biocarriers, to stem cells for tissue repair, or for tumour targeting. Several papers deal with cell tracking and imaging facilitated by superparamagnetic nanoparticles, luminescent nanocrystals, or titanium dioxide nanoparticles for dual mode enhancement of computed tomography. Another important area of regenerative medicine that is well presented in this special issue is the development of tissue engineering scaffolds. A paper by X. Wang et al. demonstrates the potential of titania-based nanostructured ceramic scaffolds to drive cell differentiation and create 
an osteogenic environment for bone tissue engineering. Novel gradient technology for high-throughput screening is proposed by A. Michelmore et al. Nanomaterials offer great promises; however, many potential health hazards are not yet understood. This special issue provides insight in this area such as the cytotoxicity of carbon nanotubes to microphages. When medical treatments are implemented (both traditional and new), infections are always a significant problem. Through a paper by L. Ploux and coworkers focused on the antibacterial properties of silver-loaded plasma polymer coatings, the special issue makes a contribution in this area too.

\section{Acknowledgments}

The editorial team would like to thank all authors for their excellent contributions. This special issue would not have been of such quality without the constructive criticism of the reviewers. We gratefully acknowledge all colleagues who contributed to the peer review process. We also acknowledge the great support and assistance of the publishing team of the Journal of Nanomaterials. K. Vasilev would also like to thank the Australian Research Council for fellowship FT100100292 which allowed him the time to edit this special issue. $\mathrm{H}$. Chen thanks the support from the Ministry of Science and Technology of China (Grant 2012CB933903). P. Murray acknowledges support from the UK Engineering and Physical Sciences Research Council (EP/H046143/1).

Krasimir Vasilev Haifeng Chen Patricia Murray 

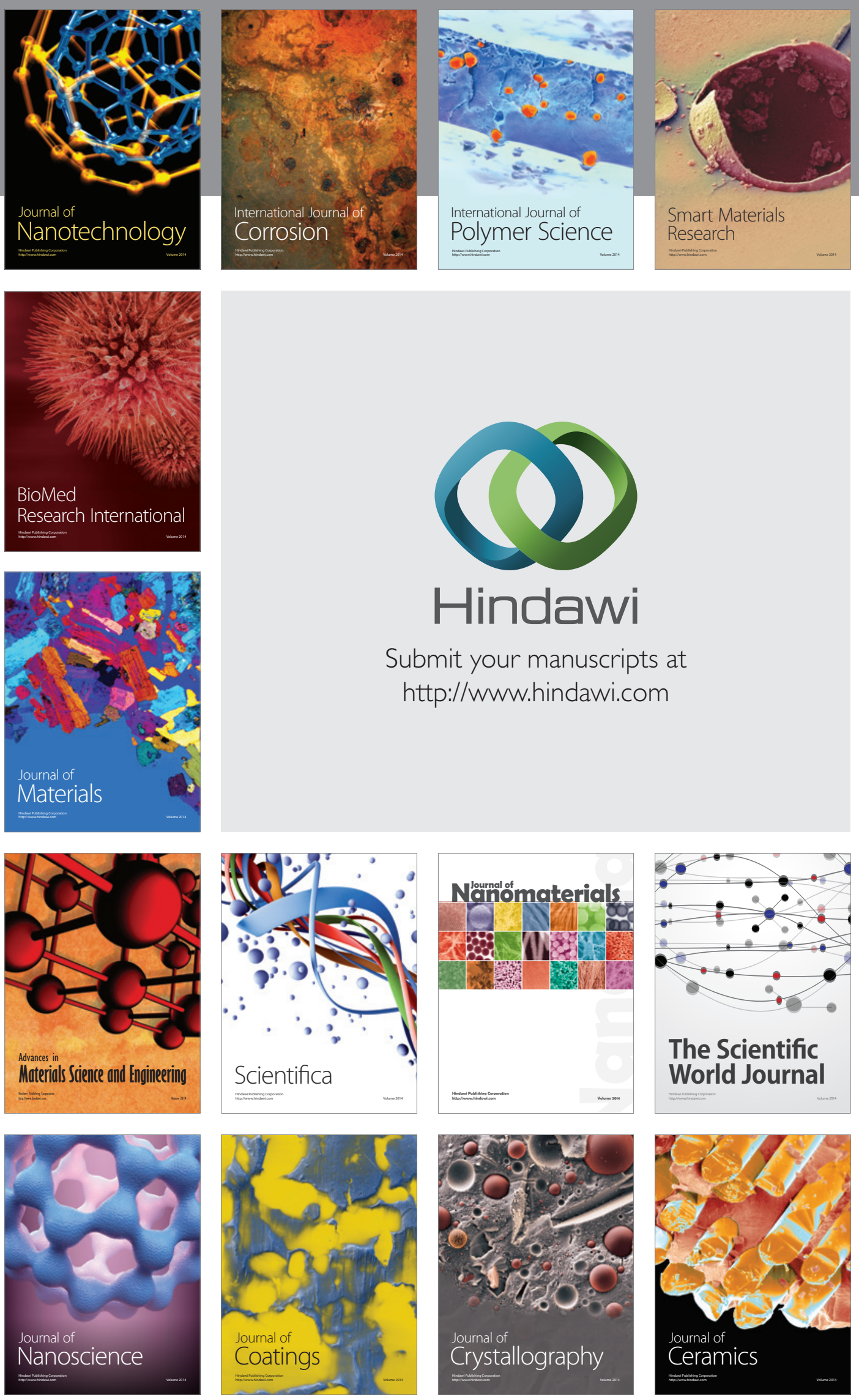

The Scientific World Journal

Submit your manuscripts at

http://www.hindawi.com

\section{World Journal}

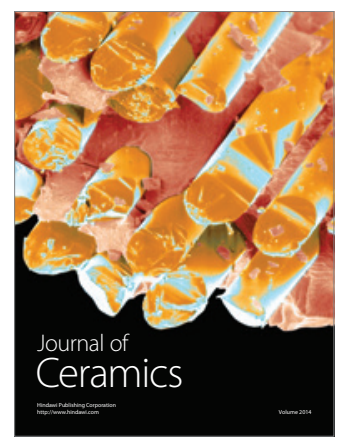

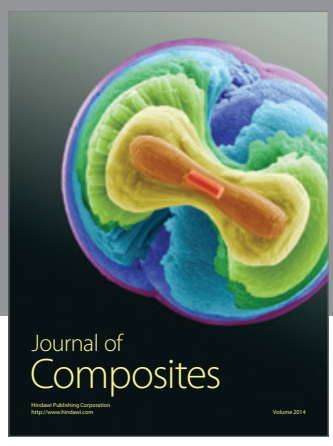
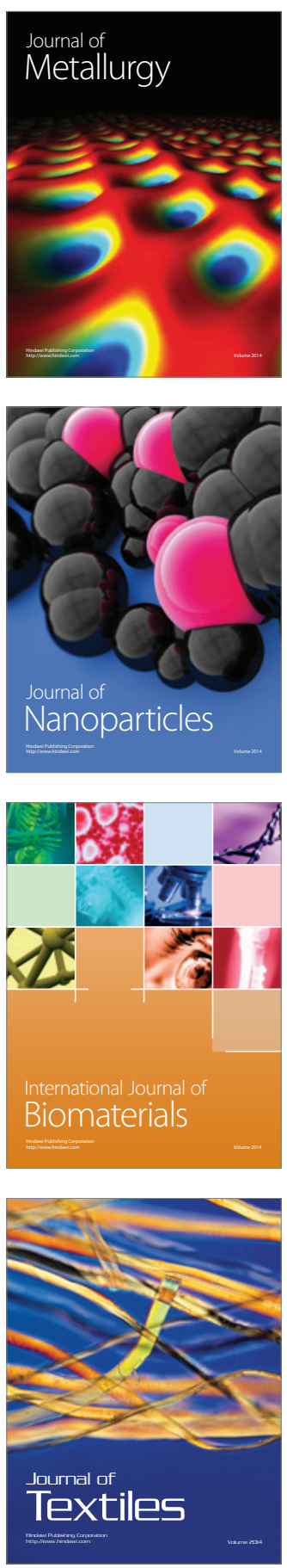